\title{
Pneumomediastinum and hyponatraemic dehydration as presenting features of cystic fibrosis
}

\section{To the Editor:}

Newborn screening for cystic fibrosis promotes early diagnosis and treatment, leading to a reduction in the number of late diagnoses [1]. This is of benefit to patients but contributes to physicians being less aware of the diverse ways in which unscreened patients present. We report a 12-year-old male who presented with pneumomediastinum and hyponatraemic dehydration, in whom subsequent investigations diagnosed cystic fibrosis. Hyponatraemic dehydration is well documented as a presenting feature of cystic fibrosis but usually occurs during periods of hot weather or after strenuous exercise [2]; neither was present in this case. Although pneumomediastinum is not uncommon in adults with advanced cystic fibrosis [3], it is very rare in children and has not previously been reported in combination with hyponatraemic dehydration.

A 12-year-old male attended the emergency department with shortness of breath, neck swelling and chest pain. The patient had been unwell for 1 week with coryza, pyrexia and a productive cough. He was dehydrated, tachycardic, tachypnoeic and hypoxic. Blood pressure was normal. He had palpable crepitus on the left side of his neck extending onto his anterior chest wall. Chest wall expansion was equal and his trachea central. On auscultation, there were coarse crackles throughout both lung fields. The only other abnormality was grade III finger clubbing.

Blood investigations confirmed dehydration and identified hyponatraemia, hypokalaemia, hypochloraemia and metabolic alkalosis. A chest radiograph (fig. 1a) confirmed subcutaneous emphysema. It also identified pneumomediastinum, hyperinflation, increased bronchovascular markings with bronchial wall thickening, dilation of perihilar airways and scattered nodular densities; there was no pneumothorax. Oxygen was initially given to treat the hypoxia, and then continued to promote "nitrogen washout" and aid pneumomediastinum absorption. He received two $20-\mathrm{mL} \cdot \mathrm{kg}^{-1}$ boluses of $0.9 \%$ saline and a dose of oral
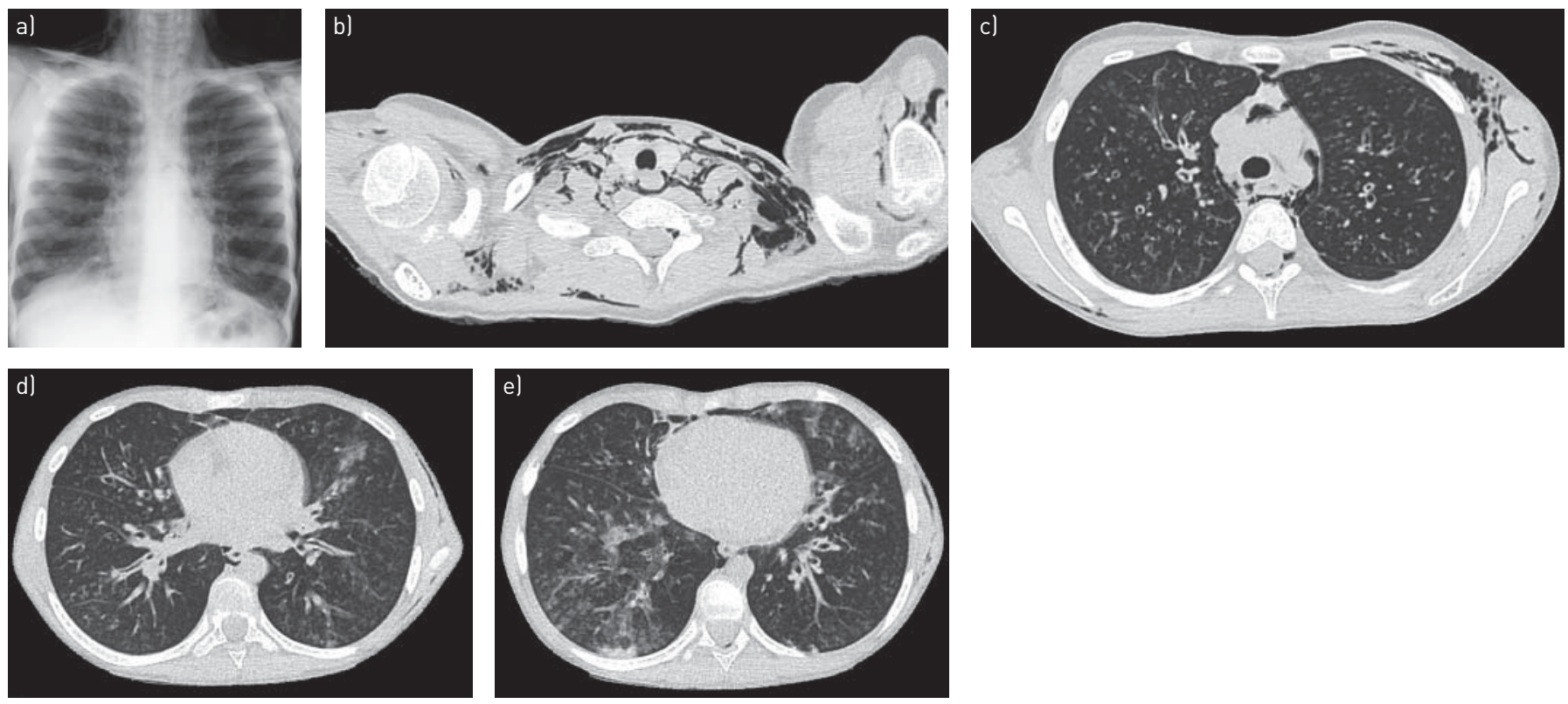

FIGURE 1 a) Chest radiograph showing extensive surgical emphysema, pneumomediastinum, bronchial wall thickening and scattered nodular densities. b) Axial computed tomography images of the chest on lung window settings, demonstrating extensive subcutaneous emphysema within the soft tissues of the thorax at the thoracic outlet. c) At the superior mediastinal level, there is left anterior chest wall subcutaneous emphysema and extensive mediastinal air. d) Within the lungs, there is bronchiectasis, bronchial wall thickening, tree-in-bud opacities and centrilobular nodules. This is most notable below the level of the hila. e) There is consolidation posteriorly in right lower lobe. 
potassium chloride. Maintenance intravenous fluids and intravenous co-amoxiclav were commenced. By day 2, his electrolyte abnormalities had almost completely normalised and his subcutaneous emphysema was no longer clinically apparent. Intravenous fluids and oxygen were therefore discontinued.

Further discussion revealed that the patient had a 1-year history of cough; this was mainly dry but he occasionally produced sputum in the morning. There was no shortness of breath or wheeze. He had a good appetite and normal bowel habit. Growth was normal (weight $37.8 \mathrm{~kg}$, 25th percentile; height $155.3 \mathrm{~cm}$, 75th percentile). Due to his cough, he had been seen by a paediatric respiratory physician 3 weeks prior to his acute admission. A number of investigations had been booked but most had not yet taken place. His urea and electrolytes from that appointment were normal.

Once clinically stable, he was fully investigated. High-resolution computed tomography of his chest (fig. 1b-e) showed widespread mediastinal air tracking into the supraclavicular fossae, the upper arms and posteriorly around the periscapular regions. No intrapleural air was seen. There was striking bronchial wall thickening, with many of the segmental airways showing mild or moderate cylindrical bronchiectatic dilatation. Peripherally, there was a profuse reticular nodularity and widespread tree-in-bud opacities reflecting small airway mucus plugging. A sweat test confirmed cystic fibrosis (chloride, $111 \mathrm{mmol} \cdot \mathrm{L}^{-1}$ ) and he was shown to be homozygous for the Phe508del cystic fibrosis transmembrane conductance regulator (CFTR) mutation. During flexible bronchoscopy, purulent secretions were seen throughout both lungs. Bronchoalveolar lavage (BAL) cultures only isolated methicillin-sensitive Staphylococcus aureus (MSSA). His i.v. antibiotics were changed to cefuroxime and flucloxacillin and he was taught airway clearance techniques by a specialised cystic fibrosis physiotherapist. Exocrine pancreas insufficiency was confirmed (faecal elastase, $<15 \mu \mathrm{g} \cdot \mathrm{g}^{-1}$ ) and the serum concentrations of fat-soluble vitamins were low: vitamin $\mathrm{A}, 0.37 \mu \mathrm{mol} \cdot \mathrm{L}^{-1}$; 25-hydroxycholecalciferol, $22.1 \mathrm{nmol} \cdot \mathrm{L}^{-1}$; and vitamin E, $6.4 \mu \mathrm{mol} \cdot \mathrm{L}^{-1}$. The patient was commenced on pancreatic enzyme replacement therapy and vitamin supplementation. The patient was discharged after a 2-week course of $i . v$. antibiotics.

The patient's 6-year-old sister was born 5 months prior to the cystic fibrosis newborn screening programme being implemented across the UK. She had a history of a productive cough and intermittent greasy stools. The day after her brother was diagnosed she attended the hospital for a sweat test, which confirmed cystic fibrosis (chloride, $102 \mathrm{mmol} \cdot \mathrm{L}^{-1}$ ). High-resolution computed tomography of her chest showed mucus plugging but no bronchiectasis and BAL cultures were positive for a MSSA and Haemophilus influenzae. She received 2 weeks of i.v. cefuroxime and flucloxacillin. The same CFTR mutations were identified, as was exocrine pancreas insufficiency. Pancreatic enzyme replacement therapy and vitamin supplements were commenced. The simultaneous, late diagnosis of cystic fibrosis in two siblings was clearly devastating news for the whole family. Significant care was taken in ensuring that the news was broken to the parents and the children in the most appropriate way. This process was led by the cystic fibrosis consultant with support from the specialist cystic fibrosis nurse and psychologist.

This is the first reported case of cystic fibrosis presenting in a child with pneumomediastinum and hyponatraemic dehydration; it highlights the variable ways in which unscreened patients can present. Patients diagnosed late are known to fare worse than patients of the same age and CFTR mutation diagnosed by newborn screening [4]. In addition, there are significant psychological consequences. The observation that a child homozygous for the Phe508del CFTR mutation and pancreatic insufficient can reach 12 years of age with relatively normal growth and few respiratory symptoms confirms that other factors can modify disease severity. These factors are likely to include diet, physical activity and polymorphisms in genes other than CFTR.

The association between cystic fibrosis and hyponatraemic dehydration was first recognised during heat wave in New York, NY, USA in 1948 [5], and led to the identification of elevated concentrations of sodium and chloride in the sweat of patients with cystic fibrosis [6]. There have subsequently been multiple reports of patients with cystic fibrosis presenting with hyponatraemic dehydration associated with hypochloraemia and metabolic alkalosis [2, 7]. Virtually all these cases have occurred during periods of hot weather but the average temperature prior to this presentation was $5^{\circ} \mathrm{C}$ and, therefore, was not the precipitant of his hyponatraemic dehydration. We hypothesise that the respiratory exacerbation and, specifically, the associated high pyrexia contributed to excess sweating and, therefore, electrolyte loss that was seen in our patient. Urinary electrolytes taken at presentation (sodium $<10 \mathrm{mmol} \cdot \mathrm{L}^{-1}$; potassium, $0 \mathrm{mmol} \cdot \mathrm{L}^{-1}$ ) confirm that the losses were not renal.

Spontaneous pneumomediastinum is defined as air in the mediastinum that appears in the absence of trauma. It is caused by alveolar rupture and dissection of air along the bronchovascular sheath into the mediastinum and hilum. A trigger factor such as the Valsalva manoeuvre or forceful coughing may be identified [8]. While spontaneous pneumomediastinum is not uncommon in adults with advanced cystic fibrosis, it is extremely rare in children. There are only two previous case reports: one in a 2-year-old female 
in whom spontaneous pneumomediastinum was the presenting feature of cystic fibrosis [9] and the other in a 10-year-old male with cystic fibrosis in whom it was the presenting feature of allergic bronchopulmonary aspergillosis [10]. Neither, however, had an associated electrolyte imbalance. We hypothesise that our patient's pneumomediastinum was caused by excess coughing due to the infective exacerbation.

In summary, this is the first reported case of pneumomediastinum and hyponatraemic dehydration as presenting features of cystic fibrosis. In addition, this case has highlighted the variable ways in which unscreened patients can present with cystic fibrosis and reminds us that despite the widespread introduction of newborn screening programmes, physicians should continue to consider cystic fibrosis a possible diagnosis in such patients. hyponatraemic dehydration http://ow.ly/pgAFQ

Francis J. Gilchrist ${ }^{1,2}$, Martin Samuels ${ }^{1}$, Gillian Klafkowski ${ }^{3}$, Nick A. Watson ${ }^{3}$ and Warren Lenney ${ }^{1,2}$

${ }^{1}$ Academic Dept of Child Health, University Hospital of North Staffordshire, Stoke on Trent, ${ }^{2}$ Institute of Science and Technology in Medicine, Keele University, Keele, and ${ }^{3}$ Dept of Radiology, University Hospital of North Staffordshire, Stoke on Trent, UK.

Correspondence: F.J. Gilchrist, Academic Dept of Child Health, University Hospital of North Staffordshire, Newcastle Road, Stoke on Trent, ST4 6QG, UK. E-mail: francis.gilchrist@uhns.nhs.uk

Received: July 222013 | Accepted after revision: Sept 062013 | First published online: Sept 262013

Conflict of interest: None declared.

\section{References}

1 Accurso FJ, Sontag MK, Wagener JS. Complications associated with symptomatic diagnosis in infants with cystic fibrosis. J. Pediatr 2005; 147: S37-S41.

2 Ballestero Y, Hernandez MI, Rojo P, et al. Hyponatremic dehydration as a presentation of cystic fibrosis. Pediatr Emerg Care 2006; 22: 725-727.

3 Flume PA. Pneumothorax in cystic fibrosis. Chest 2003; 123: 217-221.

4 Sims EJ, Clark A, McCormick J, et al. Cystic fibrosis diagnosed after 2 months of age leads to worse outcomes and requires more therapy. Pediatrics 2007; 119: 19-28.

5 Kessler WR, Andersen DH. Heat prostration in fibrocystic disease of the pancreas and other conditions. Pediatrics 1951; 8: 648-656.

6 Di Sant'agnese PA, Darling RC, Perera GA, et al. Abnormal electrolyte composition of sweat in cystic fibrosis of the pancreas; clinical significance and relationship to the disease. Pediatrics 1953; 12: 549-563.

7 Guimarães EV, Schettino GCM, Camargos PAM, et al. Prevalence of hyponatremia at diagnosis and factors associated with the longitudinal variation in serum sodium levels in infants with cystic fibrosis. J Pediatr 2012; 161: 285-289.

8 Bullaro FM, Bartoletti SC. Spontaneous pneumomediastinum in children: a literature review. Pediatr Emerg Care 2007; 23: 28-30.

9 Davies J, Chaudry R, Larovere J, et al. Cystic fibrosis presenting as acute upper airway obstruction. Thorax 2006; 61: 92 .

10 Sutrave H, Ward A, Smyth AR, et al. Pneumomediastinum as a presenting feature of allergic bronchopulmonary aspergillosis in a child with cystic fibrosis. J R Soc Med 2012; 105: Suppl. 2, S36-S39. 